\title{
SILVER nano-cylinders designed by EBL used as label free LSPR nano-biosensors
}

Neval A. Cinel Serkan Bütün Ekmel Özbay 


\title{
SILVER nano-cylinders designed by EBL used as label free LSPR nano-biosensors
}

\author{
Neval A. Cinel ${ }^{\mathrm{a},{ }^{,},}$Serkan Bütün ${ }^{\mathrm{a}}$, Ekmel Özbay ${ }^{\mathrm{a}}$ \\ a, Nanotechnology Research Center, Bilkent University, 06800 Bilkent, Turkey \\ Department of Electrical and Electronics Engineering, Department of Physics
}

\begin{abstract}
Localized Surface Plasmon Resonance (LSPR) is based on the electromagnetic-field enhancement of metallic nanoparticles. It is observed at the metal-dielectric interface and the resonance wavelength can be tuned by the size, shape, and periodicity of the metallic nanoparticles and the surrounding dielectric environment. This makes LSPR a powerful candidate in bio-sensing. In the present work, the size and period dependency of the LSPR wavelength was studied through simulations and fabrications. The surface functionalization, that transforms the surface into a sensing platform was done and verified. Finally, the concentration dependency of the LSPR shifts was observed. All the measurements were done by a transmission set-up. The study is at an early stage, however results are promising. The detection of specific bacteria species can be made possible with such a detection method.
\end{abstract}

Keywords: LSPR, EBL, biosensor, nano-cylinder, silver

\section{INTRODUCTION}

Bio-sensing is a popular topic of research ever since its importance in the rapid detection of target analytes in questionable solutions for medical diagnostics and prevention of bio-terrorism has been noticed. This popularity enabled the development of different types of biosensors relying on different transduction mechanisms. There are various optical $^{2,6}$, amperometric ${ }^{10,19}$, impedometric ${ }^{12}$, electro chemical ${ }^{20}$, and magnetic ${ }^{11,14}$ biosensors described in the literature. The sensing mechanism of these sensors is based on tracking interactions at the classical physics limit, such as measuring mass, conductivity, $\mathrm{pH}$, ionic mobility, and resonant $\mathrm{RF}$ frequency. On the other hand, sensors working at the UV-Vis-IR portion of the spectrum enable gentle detection that does not cause deterioration in the structure of matter ${ }^{13}$. When compared to other optical Surface Plasmon Resonance (SPR) ${ }^{6}$ or Surface Enhanced Raman Spectroscopy $(\mathrm{SERS})^{8,9,17}$ sensors, LSPR systems are advantageous since they are easy to manufacture, less expensive, portable and practical.

Localized Surface Plasmon Resonance (LSPR) depends on the collective oscillation of the surface conduction electrons excited by electromagnetic radiation. The resonance wavelength is related to the type of the metal used, surrounding environment, nano-particles' size, size distribution, and shape ${ }^{7}$. LSPR biosensors rely on the measurement of LSPR

wavelength shifts, caused by differences in refractive index, that are induced by the_analytes applied on the sensor surface for being detected.

\section{METHODOLOGY}

\subsection{Chemicals}

For this study, the EZ_Link Sulfo-NHS-SS-Biotin (21331) and Avidin (21121) is purchased from Pierce. 11-Amino-1undecanetiol, hydrochloride [A423-10], and 6-Hydroxy-1-Hexanethiol was purchased from Probior. Poly(methylmethacrylate) (PMMA 950 A-2) is used as resist in e-beam lithography.

*nyilmaz@ee.bilkent.edu.tr; phone 90312 290-1017; fax 90312 290-1015

Plasmonics in Biology and Medicine VIII, edited by Tuan Vo-Dinh, Joseph R. Lakowicz, Proc. of SPIE Vol. 7911, 79111I · (C) 2011 SPIE · CCC code: 1605-7422/11/\$18 · doi: 10.1117/12.879139 


\subsection{Fabrication}

LSPR is highly dependent on size, shape, period and material composition therefore precise control over these parameters is essential. There are various lithographic methods like chemical synthesis methods, nano sphere lithography (NSL), photo lithography (PL) However, E-Beam lithography (EBL) which is a modified SEM system has superior properties compared to other optical or chemical patterning techniques. EBL also enables precise placement and design of arbitrary shaped nano-particles. A large variety of geometries of various sizes, with fine features at high resolution is possible with $\mathrm{EBL}^{5}$. EBL also provides better resolution since it is not limited by diffraction limits (unlike other optical lithography methods) that end up with a resolution of around $10 \mathrm{~nm}^{18}$. This advanced precision may result at a higher cost and greater time however for academic research and development purposes this does not constitute a major problem.

Nanoparticle fabrication starts with the preparation of the sapphire substrate by spin coating Poly(methylmethacrylate) (PMMA 950 A-2), firstly at $500 \mathrm{rpm}$ for 3 seconds and then at $4000 \mathrm{rpm}$ for 40 seconds. The sample is then prebaked for 90 seconds at a hot plate heated to $180^{\circ} \mathrm{C}$ in order to evaporate the solvent in the photo resist and ends up with a harder coating. A final step of aqua-save (polymer) coating at $4000 \mathrm{rpm}$ for 40 seconds completes the preparation for EBL. After lithography with the "RAITH E-Line" system, the aqua-save is cleaned with DI water and then the sample is developed at an AZ 400K developer 1:4 for 30 seconds. After the development, the sample is cleaned in iso-propanol and blow-dried with nitrogen. The next step is the e-beam evaporation where an Ag coating of $30 \mathrm{~nm}$ is carried out in the Leybold Univex 350 Coating System. After evaporation, the samples are kept in acetone for lift off for 30 minutes, and then the excess metal is lifted off with an acetone flush onto the sample with a sterile glass injector. The final samples can be seen in Figure 1.

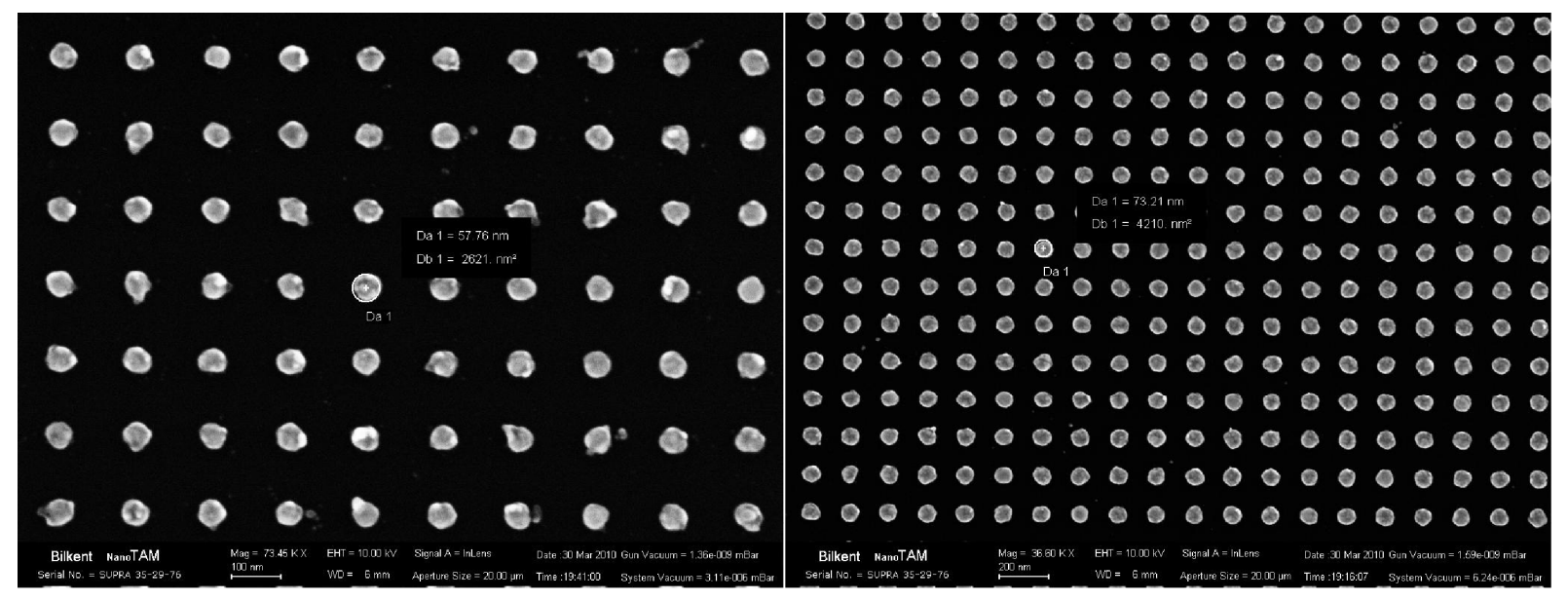

Figure 1. EBL designed nano-cylinders. EHT is set as $10 \mathrm{kV}$. Left-Diameter of nano-cylinders is about 57.76nm, Right-Diameter of nano-cylinders is about $73.21 \mathrm{~nm}$.

\subsection{Surface functionalization}

The surface functionalization step is necessary for the sensor to be specific to a particular analyte. For this aim, a twostage study has been planned. In the first stage, the sensor structure is verified with a pair of materials called biotin and avidin. As future work, the same experiment will be repeated with biotin conjugated antibody and the antigen of a specific kind of bacteria to verify that the structure can be used as a biosensor.

Avidin and biotin (Vitamin $\mathrm{H}$ ) is the most extensively studied pair in bio-sensing applications, known for one of the strongest non-covalent interactions and extraordinary affinity towards each other $\left(\mathrm{Ka}=10^{15} \mathrm{M}^{-1}\right)$. Biotin is a $244 \mathrm{Da}$ vitamin found in small amounts in all living cells and Avidin is a tetrameric protein that is usually found in egg whites ${ }^{15}$. 
The surface functionalization starts by forming a self-assembled alkanethiol monolayer (SAM) on the surface of $\mathrm{Ag}$ nano-cylinders. This is achieved by mixing $1 \mathrm{mM} 11$-amino-1-undecanethiol (11- AUT, Dojindo) and $1 \mathrm{mM}$ 6-hydroxy1-hexanethiol (6-HHT, Dojindo) 2-propanol solutions at a ratio of 1:3 for one hour. Using 6-HHT with 11-AUT reduces the non-specific adsorption and, therefore, increases the stability in the sensor response ${ }^{3}$. To remove nonspecifically adsorbed molecules after the incubation, the samples were rinsed with de-ionized water and dried with an N2 blow after the incubation. Then, for bio-tinylation, $1 \mathrm{mM}$ EZ_Link Sulfo-NHS-SS-Biotin (Pierce, 21331) was covalently linked to 11-AUT for at least three hours. Rinsing with DI water and N2 blow-drying were repeated. The samples with immobilized biotin were subjected to different concentrations of Avidin (Pierce, 21121) solutions, and real time measurements were done after rinsing and drying ${ }^{1}$. Transmission and reflection measurements are performed before and after every functionalization step.

\subsection{Simulations}

Simulations are carried out with a computer-software, which relies on "Finite-Difference Time-Domain Method". It is firstly needed to show that the LSPR phenomena can be observed in the geometry and materials of our choice and also select the best sizes for working in the desired wavelengths. In the simulations, silver nano-cylinders on sapphire with various periods and radii were used.

The results of the simulations can be seen in Figure 2 along with the dimensions of the structures. The comparison of the simulations with the experimental measurements and their explanations is provided in the Results and Discussion section.
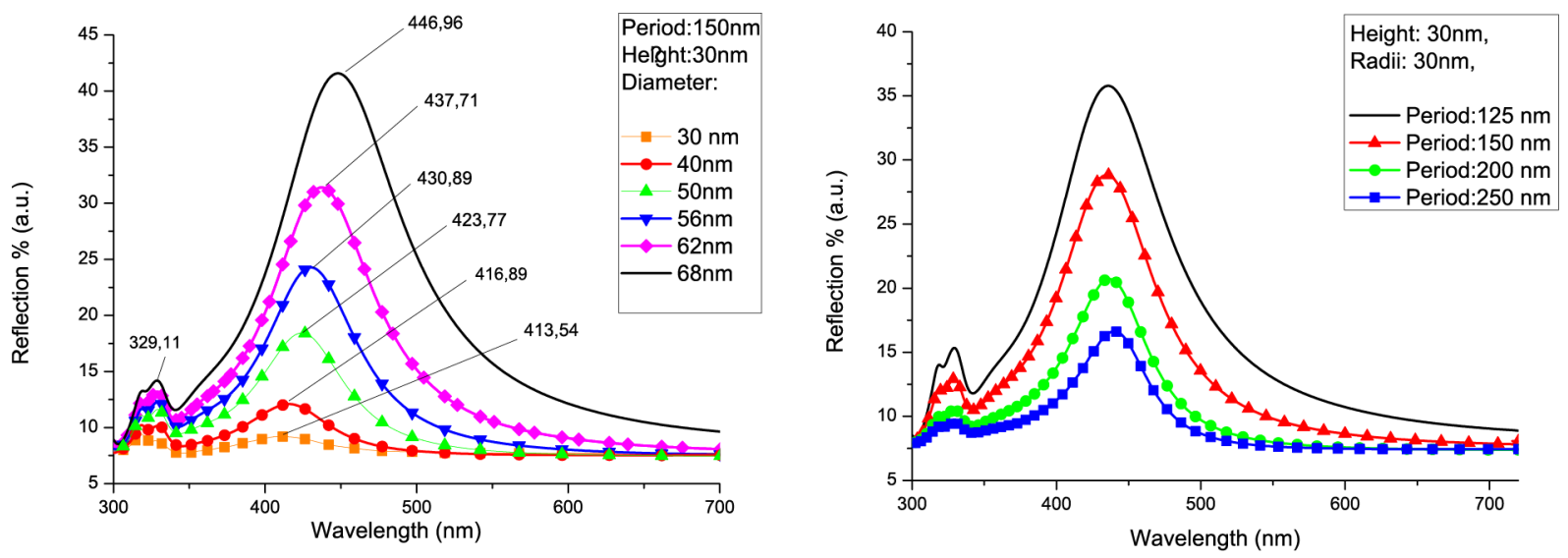

Figure 2. Reflection with respect to wavelength for silver nano-particles on sapphire. Left-Periods held constant at $150 \mathrm{~nm}$, height is $30 \mathrm{~nm}$, and the radii vary 15-34 nm. Right- Radii and the height are held constant at $30 \mathrm{~nm}$, and periods vary $150-300 \mathrm{~nm}$.

\subsection{Measurements}

In the present study, a reflection and transmission set-up has been prepared separately for the measurements.

An Ocean-Optics spectrometer, a personal computer (PC), a xenon light source (400-700nm) and an Olympus microscope with a moving stage and camera is used for taking the reflection measurements. Light from the Xenon source is transmitted through an optical fiber with a collimator at its end to the microscope and focused onto the sample using the collimating lenses of the microscope. By moving the sample stage up and down one can alter the focus of the microscope's camera using interfacing software installed on PC. The light is reflected from the sample and is collected back with an optical fiber and fed into the monochromator that diffracts it into its different frequency components. The photomultiplier of the monochromator that measures the intensity of each component is connected to the PC and the 
reflectance data is saved using special software. Graphs showing the reflection with respect to wavelength can be seen from Figure 3.

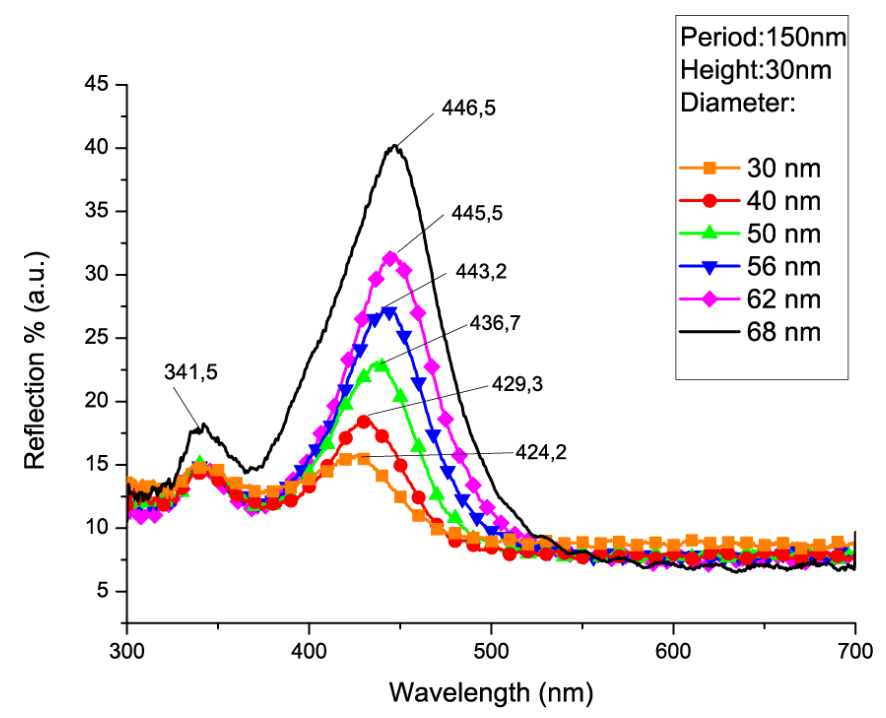

Fig. 3. Reflection measurements with respect to wavelength for silver nano-particles on sapphire. Periods held constant at $150 \mathrm{~nm}$, height is $30 \mathrm{~nm}$, and the radii vary $15-34 \mathrm{~nm}$.

The transmission measurement set-up has been prepared with an Ocean-Optics spectrometer to obtain the UV-Vis transmittance spectrum. White light of wavelength range $400 \mathrm{~nm}$ to $700 \mathrm{~nm}$ from a Xenon source is fed through a multimode optical fiber to a collimating lens that collects the light at the input and then to a lens that illuminates the biosensor. The light is transmitted through the biosensor sample and collected by a focusing lens and then a collimating lens attached to the multimode fiber.

A monochromator is used to split the light from the multimode fiber into its monochromatic components. The photomultiplier of the monochromator is connected to a personal computer and the transmission spectrum is directly available by the help of a software.

The measurement process starts with measuring the background light intensity. Than the measurement is repeated with the light source on for an empty reference point, with no nano-cylinders, and the results are saved as reference light intensity. Finally the transmitted light at the presence of the nano-cylinder arrays are measured. The transmittance is the ratio of the sample light intensity to reference light intensity, where background noise is subtracted from both measurements separately.

The transmission measurements taken from the bio samples at every step of the surface functionalization are shown in Figure 4. Outputs of the experimental measurements is discussed in the Results and Discussion section. 


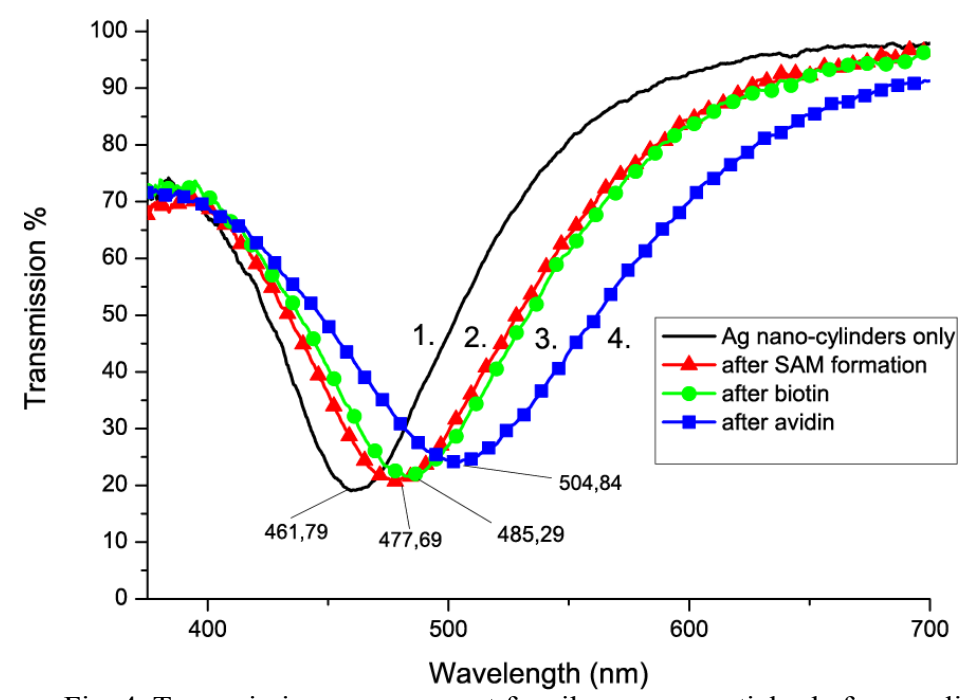

Fig. 4. Transmission measurement for silver nano-particles before application of chemicals, after the application of chemicals, after the application of biotin, and after the application of avidin.

\section{RESULTS AND DISCUSSION}

The first set of simulations and reflection measurements are in good correlation as can be seen from Figure 2 and Figure 3. In both, the increase in radius resulted in a right shift in resonance wavelength and an increase in intensity of the resonance.

In the second set of simulations, increase of the period without a change in radius, leads to a decrease in the density of the nanostructures. According to Mie Theory this should lead to a decrease in reflection intensity which is the case in the simulations where increasing the period led to a decrease in amplitude of intensity.

The LSPR wavelength shifts after every surface functionalization step can be seen in Figure 4. for the Ag nano-cylinders of the period $125 \mathrm{~nm}$, height $30 \mathrm{~nm}$, and diameter $60 \mathrm{~nm} .15 .9 \mathrm{~nm}, 7.6 \mathrm{~nm}, 19.55 \mathrm{~nm}$ red-shifts were observed after the formation of SAM, application of biotin and avidin respectively. These amounts of shifts are reasonable and sufficient to show that the binding events take place and the sensor has detected the applied material without any uncertainty.

An important feature of biosensors is their ability to determine the concentration of the analyte to be detected. Experiments have been conducted to verify that the designed sensor has different responses at different analyte concentrations and the measurements taken showed that the sensor has an adequate response at the $1 \mathrm{nM}-100 \mathrm{nM}$ range, which can be fitted to a sigmoidal curve as shown in Figure 5. Sigmoidal curves are often used for describing the specific binding of bio-molecules ${ }^{1,16,4,3}$ due to the nature of binding. This suggests that analyte binding is limited by surfacereceptor saturation and steric hindrance at high concentrations. 


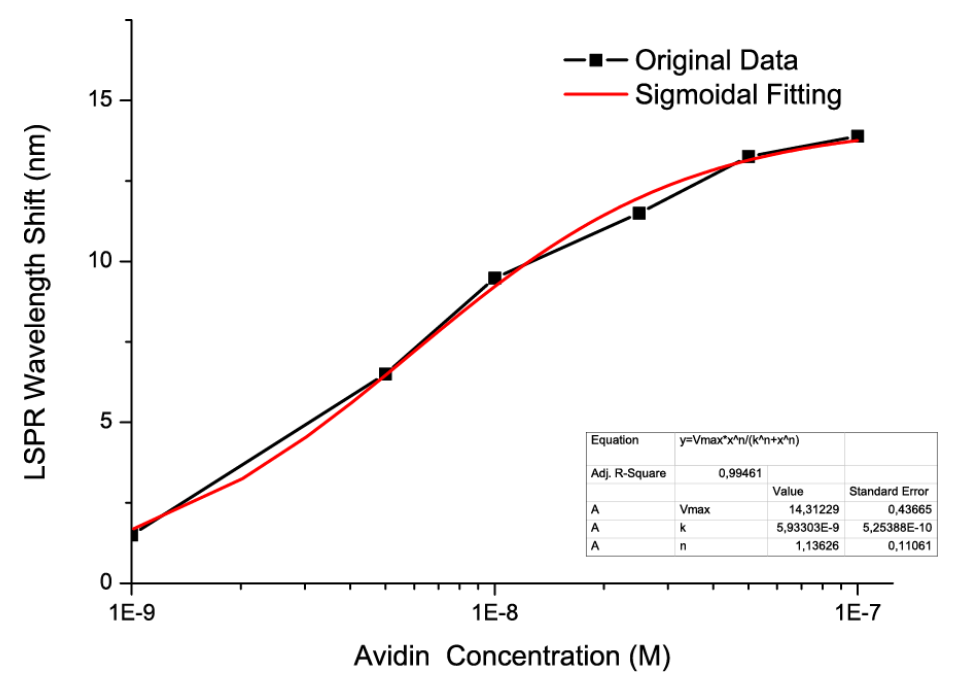

Fig. 5. LSPR Wavelength shifts with respect to avidin concentration. A sigmoidal dependence was observed and fit by hill function (red curve; inflection point slope $\approx 0.99$ ). Original data and the sigmoidal fitting are shown in the figure.

\section{CONCLUSIONS}

In this study, EBL designed silver nano-cylinders are shown to be used as label free nano-biosensors based on LSPR. Firstly, the simulations made using FDTD method helped to finalize the structures' dimensions and therefore the design. The simulations show good correlation with the measurements and the theory. EBL technique used in fabrication provides a high resolution and flexibility in patterning which helps tuning the particle's dimensions and therefore the LSPR wavelength as desired.

It is possible to extend this study for the detection of several pathogenic bacteria. Biotin which is used in the surface functionalization step is capable of being conjugated to many proteins without altering their biological activity. If biotin conjugated antibody of specific bacteria is immobilized onto the sensor then similar steps can be taken to detect the presence of its antigen in target solution. By this method, one can even achieve multi-output sensor chips that can detect several types of bacteria.

This study forms the first step of designing a multi-output sensor structure. Future work includes extended studies on modifications for detection of pathogenic bacteria. The study is at an early stage; however results are promising to proceed.

\section{ACKNOWLEDGEMENTS}

This work is supported by the European Union under the projects PHOME, ECONAM, and_N4E, and TUBITAK under the Project Nos. 109E301, 107A004, and 107A012, and DPT under the project DPT-HAMIT. One of the authors (E.O.) also acknowledges partial support from the Turkish Academy of Sciences.

\section{REFERENCES}

[1] Arai, T., Kumar, P.K.R., Rockstuhl, C., Awazu, K., Tominaga, J., "An optical biosensor based on localized surface plasmon resonance of silver nanostructured films." J Opt a-Pure Appl Op 9(7), 699-703.(2007).

[2] Cooper, M.A., "Optical biosensors in drug discovery." Nat Rev Drug Discov 1(7), 515-528.(2002).

[3] Haes, A.J., Hall, W.P., Chang, L., Klein, W.L., Van Duyne, R.P., "A localized surface plasmon resonance biosensor: First steps toward an assay for Alzheimer's disease." Nano Letters 4(6), 1029-1034.(2004). 
[4] Haes, A.J., Van Duyne, R.P., "A nanoscale optical blosensor: Sensitivity and selectivity of an approach based on the localized surface plasmon resonance spectroscopy of triangular silver nanoparticles." J Am Chem Soc 124(35), 1059610604.(2002).

[5] Hohenau, A., Ditlbacher, H., Lamprecht, B., Krenn, J.R., Leitner, A., Aussenegg, F.R., "Electron beam lithography, a helpful tool for nanooptics." Microelectron Eng 83(4-9), 1464-1467.(2006).

[6] Homola, J., Yee, S.S., Gauglitz, G., "Surface plasmon resonance sensors: review." Sensor Actuat B-Chem 54(1-2), 315.(1999).

[7] Hutter, E., Fendler, J.H., "Exploitation of localized surface plasmon resonance." Advanced Materials 16(19), 16851706.(2004).

[8] Kahraman, M., Yazici, M.M., Sahin, F., Bayrak, O.F., Culha, M., "Reproducible surface-enhanced Raman scattering spectra of bacteria on aggregated silver nanoparticles." Appl Spectrosc 61(5), 479-485.(2007).

[9] Ligler, F.S., Taitt, C.A.R., 2002. Optical biosensors : present and future, 1st ed. Elsevier, Amsterdam ; New York.

[10] Liu, X.Y., Zeng, X.D., Mai, N.N., Liu, Y., Kong, B., Li, Y.H., Wei, W.Z., Luo, S.L., "Amperometric glucose biosensor with remarkable acid stability based on glucose oxidase entrapped in colloidal gold-modified carbon ionic liquid electrode." Biosens Bioelectron 25(12), 2675-2679.(2010).

[11] Llandro, J., Palfreyman, J.J., Ionescu, A., Barnes, C.H., "Magnetic biosensor technologies for medical applications: a review." Med Biol Eng Comput2010).

[12] Malave, A., Tewes, M., Gronewold, T., Lohndorf, M., "Development of impedance biosensors with nanometer gaps for marker-free analytical measurements." Microelectron Eng 78-79, 587-592.(2005).

[13] Menon, N., "Optical biosensors:appliying photonics products to the biomedical diagnostics market," Optical Fiber Communication Conference (OFC), Los Angeles, California.(2004).

[14] Meyer, M.H., Stehr, M., Bhuju, S., Krause, H.J., Hartmann, M., Miethe, P., Singh, M., Keusgen, M., "Magnetic biosensor for the detection of Yersinia pestis." J Microbiol Methods 68(2), 218-224.(2007).

[15] Pierce, "Avidin-Biotin Technical Handbook." Thermo Scientific.(2010).

[16] Riboh, J.C., Haes, A.J., McFarland, A.D., Yonzon, C.R., Van Duyne, R.P., "A nanoscale optical biosensor: Realtime immunoassay in physiological buffer enabled by improved nanoparticle adhesion." J Phys Chem B 107(8), 17721780.(2003).

[17] Shafer-Peltier, K.E., Haynes, C.L., Glucksberg, M.R., Van Duyne, R.P., "Toward a glucose biosensor based on surface-enhanced Raman scattering." J Am Chem Soc 125(2), 588-593.(2003).

[18] Vieu, C., Carcenac, F., Pepin, A., Chen, Y., Mejias, M., Lebib, A., Manin-Ferlazzo, L., Couraud, L., Launois, H., "Electron beam lithography: resolution limits and applications." Applied Surface Science 164, 111-117.(2000).

[19] Vostiar, I., Tkac, J., Sturdik, E., Gemeiner, P., "Amperometric urea biosensor based on urease and electropolymerized toluidine blue dye as a pH-sensitive redox probe." Bioelectrochemistry 56(1-2), 113-115.(2002).

[20] Zhao, W., Xu, J.J., Chen, H.Y., "Electrochemical biosensors based on layer-by-layer assemblies." Electroanalysis 18(18), 1737-1748.(2006). 